\title{
Utilização da aprendizagem baseada em problemas (ABP) para o desenvolvimento do pensamento crítico (PC) em Matemática: uma revisão teórica
}

Resumo: A necessidade de desenvolver um pensamento crítico é uma habilidade necessária a todas a pessoas e a todos os temas. Na Matemática essa necessidade também existe, todavia, esse desenvolvimento cognitivo superior depende de uma abordagem de ensino capaz disso. No caso deste artigo o objetivo foi, por meio de ampla revisão teórica (bibliográfica e documental), determinar se a Aprendizagem Baseada em Problemas (ABP) é capaz de fomentar o desenvolvimento do pensamento matemático crítico. Segundo o estudo, diversos autores têm explorado o uso da ABP para o ensino da Matemática em assuntos específicos. Contudo, como resultado da $\mathrm{ABP}$ são percebidos incrementos, em diversas magnitudes, na capacidade cognitiva e crítica observada. Isso, por sua vez, reforça o potencial da ABP como instrumento didático seja para o ensino de Matemática, seja para o desenvolvimento do pensamento crítico dos educandos envolvidos.
Adriano Alves de Rezende

Doutorando em Educação. Professor da Universidade Estadual do Sudoeste da Bahia (UESB), campus Itapetininga. Bahia, Brasil.

(iD) orcid.org/0000-0001-9538-3080

$\triangle$ adriano.rezende01@gmail.com

Angela Ruth Silva-Salse Doutora em Educação. Professora do Doutorado em Educação da Universidad SEK e Professora da Universidad Católica del Maule (UCM). Maule, Chile.

iD orcid.org/0000-0001-9037-6834

$\triangle$ angelasilvasalse@hotmail.com

Recebido em 24/01/2021 Aceito em 11/03/2021

Publicado em 12/04/2021

Palavras-chave: Métodos de Ensino. Pensamento Crítico. Educação Matemática.

\section{Use of problem-based learning (PBL) for the development of critical thinking (CT) in Mathematics: a theoretical review}

Abstract: The need to develop critical thinking is a necessary skill for everyone and for all topics. In Mathematics this need exists. This higher cognitive development depends on a teaching approach capable of this. In the case of this article, the aim was, determines who Problem-Based Learning (PBL) is capable of fostering the development of critical mathematical thinking. Through a broad theoretical review (bibliographic and documentary). The principal results is authors explores for Mathematics teaching in specific subjects. As a result of PBL increases in many magnitudes in cognitive and critical capacities. This, in turn, reinforces the potential of PBL as a didactic tool for Mathematics teaching and for developing the critical thinking of the students involved.

Keywords: Teaching Methods. Critical Thinking. Mathematics Education.

\section{Uso del aprendizaje basado en problemas (ABP) para el desarrollo del pensamiento crítico (PC) en Matemáticas: una revisión teórica}

Resumen: La necesidad de desarrollar el pensamiento crítico es una habilidad necesaria para todas las personas y temáticas. En la Matemática esa necesidad también existe, aún más ese desarrollo cognitivo superior depende de un abordaje de enseñanza que sea capaz 
de eso. En el caso de este artículo el objetivo fue, por medio de una amplia revisión teórica (bibliográfica y documental), determinar si el Aprendizaje Basado en Problemas (ABP) es capaz de fomentar el desarrollo del pensamiento matemático crítico. Según el estudio, diversos autores han explorado el uso de la ABP para la enseñanza matemática en asuntos específicos. A partir de ello, como resultado del desarrollo de la ABP se perciben aumentos, en diversas magnitudes, en la capacidad cognitiva y crítica observada. Eso, por su parte, refuerza el potencial de la ABP como instrumento didáctico ya sea para la enseñanza de la Matemática o para el desarrollo del pensamiento crítico de los estudiantes envueltos en estos proyectos.

Palabras clave: Metodologías de Enseñanza. Pensamiento Crítico. Educación Matemática.

\section{Introdução}

Diante do atual momento de pandemia enfrentada no mundo, onde as pessoas enfrentam restrições de deslocamento e diversas atividades estão suspensas, a implementação de estratégias didáticas mais dinâmicas que favoreçam o pensamento crítico são fundamentais para manter a atenção e o comprometimento dos estudantes que se encontram cumprindo seu ano letivo por meio do ensino remoto, além de desenvolver esta habilidade necessária a todos. Tudo que antes da pandemia era facultativo agora tornou-se urgente.

Dentro deste contexto, catalisados pelo atual momento, a adequação de todas as práticas educativas ganhou mais relevância e as metodologias ativas de ensino (MAE) emergem como alternativa mais viável no processo educacional. Isso decorre principalmente pelo fato do estudante, neste tipo de abordagem didática, se tornar agente ativo do processo de aprendizagem, dentro de um contexto de dinâmica acelerada de informação e onde os instrumentos disponíveis para acessá-la estão relativamente acessíveis.

As MAE podem ser definidas como estratégias de ensino centradas na participação efetiva dos estudantes na construção do processo de aprendizagem, flexível, interligado e híbrido que se torna cada vez mais relevante dentro de um mundo dinâmico, digital e extremamente conectado (BACICHI e MORÁN, 2017). Elas contribuem de forma significativa e efetiva para a construção de soluções atuais aos anseios dos estudantes que passam de coadjuvantes para protagonistas de sua própria aprendizagem (MORÁN, 2015a; PHILLIPS, 2005). Em resumo, o estudante encontra-se no centro de tudo por meio de processos consistentes com o desenvolvimento de habilidades e não 
apenas de conteúdo.

Na Matemática, especificamente, é frequente, por parte dos alunos, o uso não consciente de procedimentos pouco eficazes, ou uso de uma técnica específica sem restrições, pois têm dificuldade de considerar outras possibilidades, o que pode implicar em resultados danosas (MASOLA e ALLEVATO, 2019).

Logo, o uso de MAE possibilita desenvolver o uso de habilidades cognitivas e físicas além do pensamento crítico e reflexivo, no intuito de fornecer ao estudante os conhecimentos necessários para enfrentar os desafios diários de sua realidade (BRUNER, 1976; GUISSO et al., 2019).

A literatura acadêmica apresenta diversas MAE como Aprendizagem Baseada em Jogos, Gamificação, Aprendizagem Baseada e Projetos, Sala de Aula Invertida, Aprendizagem por Pares, Aprendizagem Baseada em Problemas, entre outras tantas. No entanto, este artigo se dedica especificamente à Aprendizagem Baseada em Problemas (ABP ou Problem Basead Learning — PBL) pois é uma das abordagens didáticas mais relevantes para o desenvolvimento das habilidades relacionadas com a aprendizagem de Matemática.

Tal como a maioria das MAE, a ABP tem caráter interdisciplinar e segue a concepção de ensino atual bem como as definições estabelecidas na Base Nacional Comum Curricular (BNCC) e é indicada na mesma como estratégia de ação para o ensino da Matemática, Física e outras disciplinas de conteúdos que tendem a ser mais abstratos e de compreensão mais complexa.

Assim, o objetivo deste trabalho é mostrar como o uso de metodologias ativas de ensino, em específico, a Aprendizagem Baseada em Problemas (ABP) pode fomentar o desenvolvimento do pensamento crítico em estudantes nas mais diversas áreas de conhecimento, especialmente na área de Matemática.

Este artigo, além desta introdução, contém outras seções. A seguir, apresentamos os principais conceitos e abordagens referentes à Aprendizagem Baseada em Problemas $(\mathrm{ABP})$, criação de competências e o desenvolvimento do pensamento crítico (PC). Posteriormente, trazemos a descrição do método utilizado para alcançar o objetivo proposto por este estudo. Os resultados encontrados são apresentados e discutidos na seção seguinte. Na última seção são feitas as considerações finais acerca do tema. 


\section{Metodologias Ativas de Ensino (MAE)}

Já na década de 1970, Jerome Bruner (1976) defendia que os estudantes deveriam aprender a estrutura das matérias, em vez de compreender um simples conjunto de procedimentos ou fatos de forma alinhada com outras áreas do conhecimento. Segundo o autor, o desenvolvimento intelectual não é uma sequência linear de acontecimentos e que tem esse processo afetado por influências do ambiente escolar e social. Logo, o professor é partícipe fundamental desse desenvolvimento e precisa estimular os estudantes a partir da resolução de problemas que sejam desafiadores e passíveis de resolução (BRUNER, 1976). Esse pensamento se alinha perfeitamente com os preceitos das MAE e, em particular, da ABP.

As atividades desenvolvidas no modelo tradicional de ensino e na abordagem ativa possuem um denominador comum, que é o fato de ambas "aconteceram devido à forma como espaço, tempo e atividade são usados no design da disciplina" (TALBERT, 2019, p. 7). Logo, a abordagem didática empregada nas aulas é que as diferenciam. Sob esta perspectiva Brousseau (1998, p. 3) afirma que

\footnotetext{
o trabalho do professor é, em certa medida, o inverso do investigador, uma vez que ele tem de produzir uma recontextualização dos conhecimentos. Estes transformar-se-ão no conhecimento do aluno, ou seja, numa resposta bastante natural a condições relativamente particulares, condições indispensáveis para que eles tenham algum sentido para ele. Cada conhecimento tem de nascer da adaptação a uma situação específica, porque as probabilidades não se criam no mesmo gênero de contexto e de relações com o maio em que se inventa ou utiliza a aritmética ou a álgebra.
}

Assim, o uso de uma abordagem ativa de ensino por parte do professor tem que fazer sentido prático para o estudante e estar próximo de sua realidade para que este consiga absorver de forma mais fácil e definitiva o conhecimento transmitido pelo professor.

Pode-se, então, definir as MAE como estratégias de ensino embasadas na participação efetiva dos estudantes na construção do processo de aprendizagem flexível, interligado e híbrido. As metodologias ativas de ensino, num mundo dinâmico, digital e extremamente conectado, expressam-se por meio de modelos de ensino flexíveis e híbridos; contribuem de forma significativa para a construção de soluções atuais para os estudantes de hoje (BACICHI e MORÁN, 2017; MORÁN, 2015b). 
As metodologias ativas de ensino guardam em si características específicas, tal como mencionado anteriormente, que as tornam mais eficientes para alcançar os objetivos propostos por cada disciplina que as utiliza. Logo, elegeu-se a Aprendizagem Baseada em Problemas (ABP) como instrumento de fomento ao pensamento crítico (PC).

\section{Aprendizagem baseada em problemas (problem basead learning) enquanto estratégia de ensino}

A Aprendizagem Baseada em Problemas (Problem Basead Learning), propriamente dita, foi sistematizada pela primeira vez em 1969 pela universidade canadense McMaster, em seu curso de Medicina que a mantem em uso até a atualidade (LOPES et al., 2019). Ela tem sido utilizada para facilitar a compreensão de conteúdos nas mais variadas áreas de conhecimento e tem sido amplamente utilizada nas Ciências da Saúde, como em Enfermagem e Medicina seguindo os passos da Universidade McMaster.

A ABP consiste na apresentação de uma situação aos estudantes, que por sua vez, leva a um problema a ser resolvido por eles. Isso lhes permite adquirir, durante a busca pela solução destes problemas, novos conhecimentos e desenvolver novas habilidades durante o processo.

As habilidades que se busca desenvolver com o uso da ABP são: pensar e argumentar criticamente, analisar e resolver problemas complexos e reais; buscar fontes adequadas de informações, desenvolvimento da metacognição e utilizar o aprendizado obtido para continuar a aprender (DUCH, GROH e ALLEN, 2001).

A operacionalização da APB se desenvolve em ciclos e cada um, de forma sintética, se divide em três momentos distintos, tal como pode ser observado no Quadro 1 e descrito por Lopes et al., (2019) e Pinho e Lopes (2019).

Quadro 1: Momentos da aplicação do Aprendizagem Baseada em Problemas — ABP

\begin{tabular}{|c|c|c|}
\hline Etapa & Principais características & Sub etapa \\
\hline Momento 1 & $\bullet$ Construção de un problema & - Apresentação do problema. \\
$\begin{array}{c}\text { Formulação e } \\
\text { análise do } \\
\text { problema }\end{array}$ & $\begin{array}{c}\text { - Dividir a turma em grupos } \\
\text { pequenos (entre 10 a 12 }\end{array}$ & $\begin{array}{c}\text { - Identificar as informações do problema. } \\
\text { - Revisão de conhecimentos prévios. }\end{array}$ \\
\hline
\end{tabular}




\begin{tabular}{|c|c|c|}
\hline & alunos) & $\begin{array}{c}\text { - Gerar hipóteses sobre o problema. } \\
\text { - Identificar que informações faltam para } \\
\text { resolver o problema. }\end{array}$ \\
\hline $\begin{array}{c}\text { Momento 2 } \\
\text { Ciclo de }\end{array}$ & $\begin{array}{c}\text { - Aprendizagem } \\
\text { individualizada e } \\
\text { autodirigida (ou Self-direct } \\
\text { Learning - SDL) }\end{array}$ & $\begin{array}{c}\text { - Agrupar informacões relevantes para a } \\
\text { compreensão do problema (individual). } \\
\text { - Definição das estratégias a serem } \\
\text { utilizadas para a resolução do problema. }\end{array}$ \\
\hline Momento 3 & $\begin{array}{c}\text { Os alunos reúnem-se em } \\
\text { grupos novamente após } \\
\text { coleta individual de novas } \\
\text { informações para conclusão } \\
\text { do problema. }\end{array}$ & $\begin{array}{c}\text { - Compartilhamento, aplicação, debate e } \\
\text { avaliação dos novos conhecimentos até } \\
\text { que o grupo construa novas conclusões. } \\
\text { - Se o problema for satisfatoriamente } \\
\text { resolvido, o grupo redige um relatório } \\
\text { final com sua solução. }\end{array}$ \\
& $\begin{array}{c}\text { Caso isso não ocorra, um novo ciclo se } \\
\text { inicia. }\end{array}$ \\
\hline
\end{tabular}

Fonte: Baseado em Lopes et al. (2019) e Pinho e Lopes (2019)

Ressalta-se aqui a necessidade de que as equipes recebam um feedback do professor, que deverá agir como um tutor, apenas conduzindo as atividades para que os estudantes não se desviem do foco principal, a resolução do problema. Logo, como ocorre em todas as metodologias ativas de ensino, o estudante é o protagonista e, nesse caso, tende a desenvolver a habilidade de aprender a aprender.

Segundo Lopes et al. (2019) e Wood (2003), o ciclo da ABP deve se repetir quantas vezes forem necessárias até que o grupo encontre uma solução satisfatória ao problema proposto. Esses autores também entendem que ao longo de todo o processo da $\mathrm{ABP}$, os estudantes produzem registros (documentos) de suas ações, os quais o professor pode fazer uso como um instrumento de avaliação processual.

Como já mencionado, a definição do problema ou situação-problema de forma adequada é fundamental para o sucesso no uso do ABP. Assim, alguns pesquisadores como Duch, Groh e Allen (2001) e Hung (2006, 2009) desenvolveram modelos para auxiliar na elaboração das situações-problema.

O modelo de Duch, Groh e Allen (2001), por exemplo, propõem cinco etapas para construir os problemas de ABP, podendo ser resumidas da seguinte forma: $i$ ) definição de uma ideia, conceito ou princípio e relacionando-o com os objetivos de aprendizado proposto; ii) contextualização desta ideia, conceito ou princípio com o mundo real e 
desenvolvimento de problemas com uma escrita narrativa; iii) apresentação do problema com perguntas norteadoras de forma que os estudantes possam identificar os diferentes estágios do aprendizado; $i v$ ) elaboração de um guia do professor no qual se propõe uma combinação de vários contextos pedagógicos (seminários, discussões em classe, trabalhos em grupos reduzidos de estudantes etc.); v) identificação dos recursos necessários aos estudantes para operacionalizar todo seu planejamento da dinâmica da ABP.

Hung (2006) propõem inicialmente o uso do modelo 3C3R que tem seus componentes divididos em duas classes, os componentes centrais, também chamados de suporte de conteúdo e aprendizagem conceitual (3C - research, reasoning, reflection ou seja, conteúdo, contexto, conexão), e os componentes processuais (3R - research, reasoning, reflection, ou seja investigação, raciocínio e reflexão) que se referem às habilidades de processamento cognitivo e de resolução de problemas dos estudantes. Tudo isso, busca facilitar o envolvimento consciente e trazer sentido ao estudante em seu processo de aprendizado por ABP (HOLGAARD et al., 2017; PINHO e LOPES, 2019).

Para melhor compreensão das relações entres os componentes centrais e processuais Hung (2009) criou um quadro para mostrar os efeitos entre eles (Figura 1). As setas indicam a direção do efeito de um componente sobre o outro.

\begin{tabular}{|l|cc|cc|cc|}
\hline & Conteúdo & Contexto & Conexão \\
\hline Pesquisa & $\uparrow$ & $\begin{array}{c}\text { Aquisição de } \\
\text { Conhecimento }\end{array}$ & $\downarrow$ & $\begin{array}{c}\text { Direcionamento da } \\
\text { Pesquisa }\end{array}$ & $\uparrow$ & $\begin{array}{c}\text { Integração do } \\
\text { Conhecimento } \\
\text { Adquirido }\end{array}$ \\
\hline Raciocínio & $\uparrow \begin{array}{c}\text { Processamento e } \\
\text { Aplicação do } \\
\text { Conhecimento }\end{array}$ & $\downarrow$ & $\begin{array}{c}\text { Direcionamento do } \\
\text { Raciocínio }\end{array}$ & $\uparrow$ & $\begin{array}{c}\text { Integração do } \\
\text { Conhecimento } \\
\text { Adquirido }\end{array}$ \\
\hline Reflexão & $\uparrow \begin{array}{c}\text { Avaliar a Aquisição e o } \\
\text { Processamento e do } \\
\text { Conhecimento }\end{array}$ & $\uparrow$ & $\begin{array}{c}\text { Direcionamento da } \\
\text { Reflexão }\end{array}$ & $\uparrow$ & $\begin{array}{c}\text { Integração do } \\
\text { Conhecimento } \\
\text { Adquirido }\end{array}$ \\
\hline
\end{tabular}

Figura 1: Relação entre os componentes centrais e processuais do modelo 3C3R (HUNG, 2009, p. 128)

Hung (2009) propôs o uso de nove passos orientadores para a elaboração da situação-problema capaz de alcançar os objetivos didáticos e pedagógicos almejados e alinhados com o modelo 3C3R. São eles:

- Passo 1. Criar metas e objetivos - criadas metas norteadoras e objetivos de aprendizado. Devem ser traçados tanto os objetivos quanto objetivos que desenvolvam às habilidades de resolução de problemas e estudo autodirigido (Selfdirect Learning - SDL). 
- Passo 2. Conduzir uma análise de conteúdo - busca mensurar se o nível de conhecimento do estudante se mantém até o momento que estas informações sejam suficientes para que ele alcance o objetivo pedagógico proposto pelo ABP.

- Passo 3. Analisar o contexto do problema - Inserir o problema em um contexto particular visa deixá-lo mais atraente aos estudantes de modo a deixá-los mais interessados no assunto e busquem, por meio do estudo autodirigido (SDL), a solução do problema apresentado.

- Passo 4. Formular a versão inicial do problema - essencialmente, nessa fase criase um problema que seja atraente para o estudante e que o mantenha interessado e motivado. Logo, podem ser consideradas variáveis, como por exemplo: a futura profissão deles, os interesses pessoais, situações polêmicas, problemas locais ou regionais, sociais e econômicos.

- Passo 5. Conduzir uma análise de adequação do problema - essa análise é descritiva tem três seções: $i$. entender o problema; $i i$. processos de resolução do problema; e iii. domínio do conhecimento necessário para solucionar o problema. Nesse ponto é importante identificar quais são os conhecimentos centrais para a resolução do problema e quais são os periféricos.

- Passo 6. Conduzir uma análise de correspondência - Esse passo visa garantir a confiabilidade e efetividade do problema para alcançar seus objetivos de aprendizagem, ajudando a detectar se a situação-problema elaborada corresponde à cobertura de conteúdo pretendida e ao nível de habilidades dos estudantes. Logo, revisam-se os componentes conteúdo, pesquisa, raciocínio e contexto do problema utilizando as informações do Quadro 2 para visualizar a adequação do problema diante de cada componente.

- Passo 7. Conduzir processos de calibração - nesse momento os problemas identificados na fase anterior são corrigidos, removendo ou acrescentando informações à situação-problema de forma a tornar os objetivos de aprendizagem propostos mais claros e precisos quanto possível bem como manter o comprometimento do estudante em participar em seu processo de aprendizado.

- Passo 8. Construir componentes reflexivos - essa fase é especialmente importante na elaboração de problemas a serem desenvolvidos em cursos nos quais a ABP é 
um processo integrado do currículo acadêmico e os participantes possuem conhecimento prévio da ABP. A elaboração destes componentes ajuda no desenvolvimento de habilidades de estudo autodirigido dos estudantes.

- Passo 9. Examinar relações de suporte entre os componentes $3 C 3 R$ - consiste em verificar o ajustamento dos componentes 3C3R, observando se estão contidos e se ocorre a interrelação entre eles. O propósito desse último passo é garantir a criação de uma situação-problema que seja eficaz naquilo que se propõe: ensinar e estimular a autonomia do estudante em seu próprio processo de aprendizagem.

Todavia, ressalta-se que estes modelos são apenas orientações norteadoras e sua utilização não guarda qualquer tipo de relação de causalidade como o sucesso na aplicação dos modelos de ABP junto aos estudantes no que tange à aprendizagem, dado que existem outras variáveis nesse processo. Mas, eles certamente diminuem o insucesso e erros ao longo do processo de criação do problema a ser tratado.

Segundo Marques (2008), a problematização, em si, tem como objetivo desenvolver o espírito crítico, tido como base da autonomia. Normalmente é aplicado ao ensino dos valores, pois consiste no questionamento da sua legitimidade ou verdade, colocando lado a lado os vários ângulos e as várias perspectivas do problema. Esta estratégia determina que para cada objetivo temático se apresentem várias questões e alguns problemas que levem o estudante a confrontar várias possibilidades de solução. Parte do pressuposto de que o estudante é o construtor do seu próprio conhecimento.

Tão importante quanto a criação da situação problema é envolver os estudantes no processo de aprendizagem. Nesse sentido Torp e Sage (2002) e Wood (2003) destacam que na Aprendizagem Baseada em Projetos, o estudante é agente participante do processo. A ABP trabalha ativamente com complexidade da situação, pesquisando e resolvendo os problemas como parte envolvida no processo de solução. Nesse mesmo sentido, estes autores afirmam que os estudantes acabam desenvolvendo habilidades cognitivas sintetizam e constroem o conhecimento necessário para obter à solução dos problemas de forma a questionar condições que eles mesmos criaram - e metacognitivas - estratégias particulares para possibilitar e direcionar a própria aprendizagem - ao longo desse processo, afirmações que são corroboradas por Lima e Linhares (2008). 


\section{$4 O$ pensamento matemático crítico enquanto competência}

Para Villarini Jusino (2003), o pensamento crítico permite a cada pessoa examinar sua coerência, fundamentação, interesses e valores. Ou seja, um pensamento que se volta para si mesmo. Já Skovsmose (2008) define pensamento crítico como o desenvolvimento da criticidade nos indivíduos, mas para isso faz-se necessário o afloramento de capacidade individual de analisar e buscar alternativas para solucionar conflitos ou crises ocorridas diariamente. Isso também pode e deve ser construído por meio do ensino de Matemática tal como preconizado por Skovsmose $(2007,2008)$ e D’Ambrósio (1996).

Villarini Jusino (2003) propôs um modelo para entendimento e análise do pensamento crítico no qual o crescimento, eficácia e criatividade do pensamento repousam em sua capacidade contínua de autoexame, autocrítica e autocontrole que proporcionam a capacidade crítica ao indivíduo, no caso, os estudantes, a partir de metacognição (Figura 2). Essa argumentação por sua vez também se assemelha ao entendimento de Lipman (1988) bem como os pensamentos preconizados por Guzzo e Lima (2018), Siegel (1988) e Skovsmose (2008) sobre pensamento crítico.

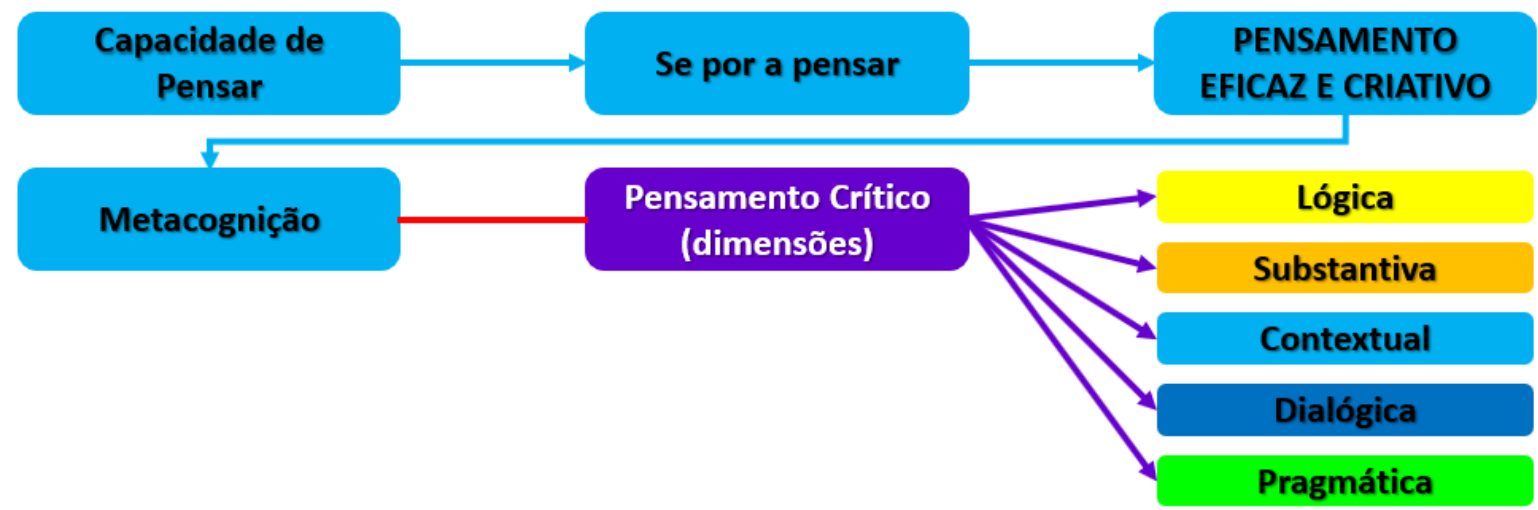

Figura 2: Desenvolvimento do pensamento crítico (Adaptado de Villarini Jusino, 2003, p. 39)

Villarini Jusino (2003) define pensamento crítico como a capacidade do pensamento para examinar e auto evoluir - com base nos próprios pensamentos ou de outros - e sendo norteado por cinco dimensões norteadoras, tal como demonstradas na Figura 2. São elas:

1. Lógica - capacidade para examinar em termos claros os conceitos e a coerência e validade do processo de raciocínio que se leva a cabo conforme as regras estabelecidas pela lógica.

2. Substantiva - a capacidade para examinar em termos de informação, conceitos, métodos ou modos de conhecer a realidade que se possuem e que se derivam de diversas disciplinas (nas quais representam o 
conhecimento que se tem como objetivo e válido).

3. Contextual - capacidade para examinar a relação com o conteúdo bibliográfico e social no qual se leva a cabo a atividade de pensamento e do qual é uma expressão.

4. Dialógica - capacidade para se examinar com relação ao pensamento dos outros, para assumir outros pontos de vista e para mediar entre diversos pensamentos.

5. Pragmática - a capacidade de examinar, em termos dos fins e interesses que busca o pensamento e das consequências que produz; analisar as lutas de poder ou as paixões a que responde o pensamento (VILLARINI JUSINO, 2003, p. 39-40).

Skovsmose (2007) defende a ideia de um ensino de Matemática que valorize a Educação Matemática Crítica de modo que o ensino forneça aos estudantes instrumentos que os auxiliem, não apenas na análise de uma situação crítica, mas também na busca por alternativas para resolver desta situação. Nesse sentido, deve-se não somente ensinar aos estudantes a usar os mais variados modelos matemáticos, mas antes levá-los a questionar todos os parâmetros que balizam sua utilização — Porquê? Como? Para quê? Quando utilizá-los?.

\section{Metodologia}

Esta é uma pesquisa qualitativa e tem caráter indutivo. Como qualitativa, busca a partir da análise de documentos, textos, fotos e imagens similaridades de significados subjetivos, dar significado à experiência da prática cotidiana e suas interrelações por meio da análise de suas experiências relatadas ou suas interrelações (FLICK, 2009; GIBBS, 2012,). Apresenta caráter indutivo pois geram e justificam uma explicação geral fundamentada no acúmulo de muitas diversas particulares, mas que se assemelham (GIBBS, 2012).

Assim, foi realizado com base em uma pesquisa bibliográfica exploratória ampla em artigos científicos, teses e dissertações incialmente brasileiras, na Biblioteca Digital Brasileira de Teses e Dissertações (BDTD) e Portal de Periódicos da Capes, e posteriormente na América Latina, no intuito de embasar o entendimento de que, se usado corretamente, a APB pode fomentar o desenvolvimento do pensamento crítico junto aos educandos. Esse procedimento encontra respaldo no trabalho de Cardoso (2017) que realizou estudo semelhante. 
A busca baseou-se no assunto estudado, ou seja, pesquisou-se termos como: pensamento crítico (PC), Aprendizagem Baseada em Problemas (ABP) e estratégias de ensino. Posteriormente, após leitura dos trabalhos selecionados foi feita uma análise qualitativa de seus conteúdos a fim de fundamentar as conclusões apresentadas.

\section{Resultados}

Inicialmente observou-se que durante a busca na BDTD, que considerou o período de seis anos (2015 a 2020), nos anos de 2017 e 2018 houve maior resultado aos termos pesquisados, sejam elas teses, sejam dissertações.

Duch, Groh e Alle (2001) enfatizam que a ABP busca desenvolver habilidades como: $i$. pensar e argumentar criticamente, bem como ser capaz de analisar e resolver problemas complexos e reais; $i i$. buscar informações em fontes apropriadas; $i i i$. saber se expressar sobre o quê e quanto aprendeu; $i v$. utilizar o aprendizado obtido para buscar sempre aprender mais. Isso tende a tornar o estudante um agente ativo do processo de aprendizagem e o leva a refletir criticamente sobre problemas reais e propondo soluções factíveis.

Diversos estudos abordam o uso da ABP na aprendizagem nas mais variadas áreas do conhecimento que são mais tangíveis aos olhos do estudante (ex.: Biologia e Pedagogia), até de conteúdos mais densos e abstratos como as da área de Ciências Exatas (ex.: Estatística, Matemática, Física, Química) e que obtêm a mesma eficiência, desde que aplicado adequadamente. Isso mostra a amplitude de emprego da ABP como ferramenta didática.

Outras obras, como a de Morales Bueno (2018), Dos Santos (2018), Barbosa (2018), Pazán e Flores (2019), Oliveira et al. (2020); Guisso et al. (2019) desenvolveram pesquisas utilizando a $\mathrm{ABP}$ voltadas para analisar a capacidade do método de fomentar a habilidade de pensamento ordenado superior, no caso, o pensamento crítico ou do seu uso para o efetivo desenvolvimento do pensamento crítico. Todos estes autores chegaram ao entendimento de que a $\mathrm{ABP}$ é capaz de desenvolver o pensamento crítico além de possibilitar que os indivíduos desenvolvam competências que lhes permitam ser mais atuantes em suas atividades e na sociedade onde estão inseridos.

O trabalho de Morales Bueno (2018) também pesquisa a aplicabilidade do ABP 
para desenvolver habilidade de pensamento ordenado superior, no caso o pensamento crítico. O autor vale-se para a análise de aspectos da abordagem ABP que podem influenciar o nível de desempenho durante a busca pelo aprimoramento dessas habilidades no ensino universitário. Além de Morales Bueno (2018), as teses doutorais de Cortez (2018), Dos Santos (2018) e a dissertação de mestrado de Barbosa (2018) também abordam o uso do ABP para o desenvolvimento do pensamento crítico.

A pesquisa de Souza (2019), no entanto, utiliza ABP em problemas matemáticos para o ensino de Cálculo Diferencial e Integral orientados a resolução de problemas reais, factuais e pertinentes as carreiras dos universitários pesquisados. Tem-se também nessa situação o afloramento do pensamento crítico e reflexivo decorrente da abordagem ABP.

Blass e Irala (2020) investigaram as possibilidades e as limitações ao longo do processo de implementação da metodologia da ABP no ensino de Cálculo Numérico. Os autores concluíram que o uso da PBL gerou aulas mais dinâmicas, maior interesse dos alunos, estimulou o estudo individual, a relação professor-estudante se tornou mais cooperativa e houve evidente construção de significados do conteúdo matemático explorado.

Torp e Sage (2002), Wood (2003) e Lima e Linhares (2008) destacaram que na ABP o estudante trabalha ativamente com a complexidade da situação, pesquisando e resolvendo os problemas como parte do processo de solução. Logo, os autores afirmam que os estudantes acabam desenvolvendo habilidades cognitivas (sintetizam e constroem o conhecimento necessário para obter à solução dos problemas de forma a questionar condições que eles mesmos criaram) e metacognitivas (estratégias particulares para possibilitar e direcionar a própria aprendizagem) ao longo desse processo.

Destaca-se que todo esse processo descrito por esses autores é necessário para o desenvolvimento do pensamento crítico, tal como afirmado por Villarini Jusino (2003), Manrique e Troussel (2014), Florez e Sandoval (2015) e Skovsmose (2007, 2008).

De maneira geral, diversos estudos tratam da aplicação da abordagem ABP para a aprendizagem de disciplinas mais densas e de conteúdo abstrato e corroboram sua eficiência, como é o caso de Matemática, desde que o método tenha sido adequadamente aplicado.

Existem diversos outros trabalhos onde a abordagem ABP é empregada nas mais 
variadas áreas do conhecimento, dentre os quais pode-se citar o de Pazán e Flores (2019) que trabalham o uso do ABP com o objetivo de fomentar os estudantes de Engenharia de Qualidade a pensar criticamente e desenvolver capacidades - comunicação oral, comunicação escrita e aprendizagem autônoma — que lhes permitam ser mais ativos em sua profissão e na sociedade.

Oliveira et al. (2020) elaboraram uma pesquisa de caráter bibliográfico sobre o uso de metodologias ativas, em particular a $\mathrm{ABP}$, e principalmente na prática curricular de Pedagogia. Os autores concluíram que o uso da ABP na prática curricular de um curso de Pedagogia possibilita uma abordagem metodológica que na formação docente utiliza o trabalho em equipe e a construção de pensamento crítico.

Andreatta e Allevato (2020) realizaram um estudo tentando compreender como problemas elaborados por estudantes do $5^{\circ}$ ano do Ensino Fundamental de uma Escola Municipal Comunitária Rural poderiam contribuir para suas próprias aprendizagens. Os autores identificaram que os problemas elaborados pelos estudantes estiveram relacionados, em sua maior parte, às operações fundamentais da Matemática prioritariamente adição e multiplicação -, mas apresentam grande potencial para desenvolver neles aspectos de criatividade e de criticidade.

Guisso et al. (2019) debateram as características da Aprendizagem Baseada em Problemas (ABP) para utilização no Ensino Superior. Para os autores, a ABP promove a habilidade de trabalhar em grupo, a racionalização crítica do ambiente organizacional, estimula o estudo individual e é centrada no estudante, tornando-o o maior responsável por seu aprendizado. O uso da ABP potencializa a formação das características necessárias aos profissionais da sociedade atual que necessitam desenvolver o raciocínio lógico e o pensamento crítico são exigências do mercado de trabalho. Essa abordagem metodológica também é identificada por Godoy e Almeida (2017) em levantamento realizado em trabalhos que discutem o ensino de Matemática em cursos de Engenharia.

Por sua vez, Mendes da Silva, Linz e Carneiro Leão (2019) fizeram uso da ABP na disciplina de Tecnologia da Informação e Comunicação (TIC) no Ensino de Química (TICEQ) no intuito de analisar se ela proporciona aos estudantes um processo formativo mais crítico quanto às discussões sobre a integração das TIC no ensino de Química. Os resultados encontrados foram similares aos encontrados nos trabalhos de Morales Bueno 
(2018), Dos Santos (2018), Barbosa (2018), Pazán e Flores (2019), Oliveira et al. (2020) e Guisso et al. (2019).

Todavia, Marques (2008) ressalta que o método de problematização não é unanimidade, pois recebe críticas de vários autores que questionam a excessiva problematização deste método, o qual nem sempre cuida de que os estudantes tenham tido uma oportunidade prévia de se identificarem com modelos, exemplos, costumes e cultura.

Outras pesquisas sobre o desenvolvimento do Pensamento Matemático Crítico por meio da ABP, seja de forma direta e indireta, encontram-se apresentadas no Quadro 2.

Quadro 2: Pesquisas recentes sobre o uso da Aprendizagem Baseada em Problemas para o ensino de Matemática e Desenvolvimento do Pensamento Crítico

\begin{tabular}{|c|c|c|}
\hline Autores & Título & Objetivo \\
\hline $\begin{array}{c}\text { Jerônimo, } \\
\text { Zanette e } \\
\text { Schneider (2019) }\end{array}$ & $\begin{array}{l}\text { A Matemática na Educação a } \\
\text { Distância: possibilidade de uso de } \\
\text { Metodologias Ativas de } \\
\text { Aprendizagem }\end{array}$ & $\begin{array}{c}\text { Investigar os processos de ensino e } \\
\text { aprendizagem de Matemática na } \\
\text { Educação a Distância }(\mathrm{EaD}) \text { com o } \\
\text { uso de metodologias ativas de } \\
\text { aprendizagem }\end{array}$ \\
\hline $\begin{array}{c}\text { Coelho e } \\
\text { Malheiro (2019) }\end{array}$ & $\begin{array}{c}\text { Habilidades cognitivas em } \\
\text { processos formativos de professores } \\
\text { da Educação Básica na } \\
\text { Aprendizagem Baseada em } \\
\text { Problemas }\end{array}$ & $\begin{array}{c}\text { O objetivo foi analisar os níveis de } \\
\text { habilidades cognitivas } \\
\text { desenvolvidos por uma equipe de } \\
\text { seis docentes ao solucionarem um } \\
\text { problema autêntico, proposto por } \\
\text { eles, ao longo de um Curso de } \\
\text { Férias desenvolvido na } \\
\text { Universidade Federal do Pará } \\
\text { (UFPA) }\end{array}$ \\
\hline $\begin{array}{l}\text { Díaz, Polo e } \\
\text { Toro (2017) }\end{array}$ & $\begin{array}{l}\text { Metodologías activas para la } \\
\text { solución de problemas al enseñar } \\
\text { Matemáticas Financieiras }\end{array}$ & $\begin{array}{c}\text { O objetivo desta pesquisa era } \\
\text { analisar o impacto da } \\
\text { implementação da Aprendizagem } \\
\text { Baseada em Problemas (ABP) no } \\
\text { ensino de Matemática Financeira }\end{array}$ \\
\hline Romero (2019) & $\begin{array}{c}\text { Propuesta de actividades mediante } \\
\text { la metodología ABP para la } \\
\text { conceptualización del cálculo } \\
\text { integral }\end{array}$ & $\begin{array}{c}\text { Desenvolver uma proposta didática } \\
\text { com a ABP tornar o ensino de } \\
\text { Cálculo Integral, para estudantes de } \\
\text { Comércio Exterior da Universidad } \\
\text { de Las Américas (Quito, Equador), } \\
\text { tornando-os mais participativos e } \\
\text { proativos }\end{array}$ \\
\hline $\begin{array}{l}\text { Muñoz Choque } \\
\text { (2019) }\end{array}$ & $\begin{array}{l}\text { Desarrollo de competencias } \\
\text { financieras a través de la técnica de }\end{array}$ & $\begin{array}{l}\text { Avaliar o efeito da técnica de } \\
\text { resolução de problemas para o }\end{array}$ \\
\hline
\end{tabular}




\begin{tabular}{|c|c|c|}
\hline & $\begin{array}{l}\text { resolución de problemas en los } \\
\text { estudiantes de la Facultad de } \\
\text { Contabilidad de la Universidad de } \\
\text { Ciencias y Humanidades }\end{array}$ & $\begin{array}{l}\text { desenvolvimento de competências } \\
\text { financeiras nos estudantes de } \\
\text { especialização em Contabilidade da } \\
\text { Universidad de Ciencias y } \\
\text { Humanidades (Lima, Peru), no ano } \\
\text { de } 2016\end{array}$ \\
\hline $\begin{array}{l}\text { Viana e Lozada } \\
\quad(2020)\end{array}$ & $\begin{array}{c}\text { Aprendizagem baseada em } \\
\text { problemas para o ensino de } \\
\text { probabilidade no Ensino Médio e a } \\
\text { categorização dos erros } \\
\text { apresentados pelos alunos }\end{array}$ & $\begin{array}{l}\text { A pesquisa teve como objetivo foi } \\
\text { verificar a efetividade da } \\
\text { metodologia ativa de Aprendizagem } \\
\text { Baseada em Problemas (ABP) no } \\
\text { ensino de Probabilidade no Ensino } \\
\text { Médio, com vistas a desenvolver as } \\
\text { competências e habilidades } \\
\text { previstas na Base Nacional Comum } \\
\text { Curricular. }\end{array}$ \\
\hline $\begin{array}{c}\text { Mulyanto, } \\
\text { Gunarhadi e } \\
\text { Indriayu (2018) }\end{array}$ & $\begin{array}{l}\text { The effect of problem based } \\
\text { learning model on student } \\
\text { mathematics learning outcomes } \\
\text { viewed from critical thinking skills }\end{array}$ & $\begin{array}{l}\text { Este estudo tem como objetivo } \\
\text { determinar o efeito da aplicação de } \\
\text { um modelo de aprendizagem } \\
\text { baseado em problemas nos } \\
\text { resultados de aprendizagem de } \\
\text { Matemática dos estudantes, vistos a } \\
\text { partir das habilidades de } \\
\text { pensamento crítico de estudantes do } \\
\text { quinto ano da escola primária } \\
\text { privada na região de Surakarta, } \\
\text { Java, Indonésia }\end{array}$ \\
\hline Aini et al. (2019) & $\begin{array}{l}\text { Problem-Based Learning for } \\
\text { Critical Thinking Skills in } \\
\text { Mathematics. }\end{array}$ & $\begin{array}{c}\text { Este estudo tem como objetivo } \\
\text { descobrir como desenvolver o } \\
\text { pensamento crítico de um estudante } \\
\text { habilidades por meio da } \\
\text { aprendizagem baseada em } \\
\text { problemas }\end{array}$ \\
\hline $\begin{array}{c}\text { Maulidiya e } \\
\text { Nurlaelah (2019) }\end{array}$ & $\begin{array}{l}\text { The effect of problem-based } \\
\text { learning on critical thinking ability } \\
\text { in mathematics education. }\end{array}$ & $\begin{array}{c}\text { Os objetivos estabelecidos no } \\
\text { estudo foram examinar a } \\
\text { capacidade de o pensamento crítico } \\
\text { dos estudantes, a atividade deles e a } \\
\text { capacidade dos professores em } \\
\text { gerenciar o processo de } \\
\text { aprendizagem usando a } \\
\text { aprendizagem baseada em } \\
\text { problemas }\end{array}$ \\
\hline
\end{tabular}

Fonte: Elaboração dos Autores, 2021

Ao observar os trabalhos contidos no Quadro 2 percebe-se que, por meio do uso da $\mathrm{ABP}$, os estudantes foram estimulados a desenvolver diversos sentidos, habilidades e competências que, em maior ou menor grau, impactam no seu processo de autoconhecimento e evolução como indivíduo e profissional, sendo necessário, conforme 
demonstrado em alguns trabalhos, adequações para maior efetividade do método.

\section{Conclusões}

Com base na revisão de literatura realizada foi possível constatar que a ABP é passível de aplicação em uma diversidade de temáticas e áreas de conhecimento, podendo ser efetivamente aplicada no ensino dos conteúdos de Ciências Exatas, em particular da Matemática, com o propósito de desenvolver o pensamento crítico, desde que o problema e as perguntas norteadores sejam bem elaboradas e o objetivo pedagógico sejam bem definidos.

Por ser uma abordagem ativa com caráter interdisciplinar, a ABP leva os estudantes, participantes do processo, a desenvolver o pensamento crítico, o raciocínio lógico e competências para se tornarem indivíduos mais atuantes e plenos, não só em suas vidas, mas também dentro da sociedade onde se inserem. Todavia, cabe ressaltar que ficou patente a necessidade de adequações as diversas realidades observadas, antes do emprego do método, para sua maior efetividade como instrumento pedagógico capaz de desenvolver o pensamento crítico não só na Matemática, mas em todas as áreas de conhecimento e nos mais variados níveis de ensino.

Logo, entende-se que o objetivo proposto foi alcançado. Assim, acredita-se que a Aprendizagem Baseada em Problemas seja, além de um significativo instrumento de apoio ao ensino, constitui-se um método capaz de fomentar o pensamento matemático crítico.

\section{Referências}

AINI, Nur Rohmatul; SYAFRIL; Syafrimen; NETRIWAT; Netriwati; PAHRUDIN, Agus; RAHAYU, Titik; PUSPASARI, Vinda. Problem-Based Learning for critical thinking skills in Mathematics. Journal of Physics: Conference Series, p. 1-8, 2019.

ANDREATTA, Cidmar; ALLEVATO, Norma Sueli Gomes. Aprendizagem matemática através da elaboração de problemas em uma escola comunitária rural. Educação Matemática Debate, Montes Claros, v. 4, n. 10, p.1-28, 2020.

BACICHI, Lilian; MORÁN, José. Metodologias ativas para uma educação inovadora: uma abordagem teórico-prática. São Paulo: Penso, 2017.

BARBOSA, Elen Aleixo. O uso da metodologia da problematização para o 
desenvolvimento do pensamento crítico. 2018. 216f. Dissertação (Mestrado em Educação) — Centro Universitário Adventista de São Paulo. Engenheiro Coelho.

BLASS, Leandro; IRALA, Valesca Brasil. O uso da Aprendizagem Baseada em Problemas (PBL) como metodologia de ensino em aulas de Cálculo Numérico. Revista de Educação Matemática, v.17, p. 1-24, 2020.

BROUSSEAU, Guy. La théorie des situations didactiques en Mathématiques. Grenoble: La Pensée Sauvage,1998.

BRUNER, Jerome Seymour. O processo da Educação. Tradução de Lólio Lourenço de Oliveira. São Paulo: Nacional, 1976.

CARDOSO, Marcus Algusto Martins. Transposição e ressignificação das metodologias ativas para o Ensino Médio, à luz das políticas educacionais brasileiras. 2017. 96f. Dissertação (Mestrado em Políticas Públicas e Desenvolvimento Local) — Escola Superior de Ciências da Santa Casa de Misericórdia de Vitória. Vitória.

COELHO, Antônia Ediele de Freitas; MALHEIRO, João Manoel da Silva. Habilidades cognitivas em processos formativos de professores da educação básica na aprendizagem baseada em problemas. Investigações em Ensino de Ciências, Porto Alegre, v. 24, n. 2, p. 163-180, maio/ago. 2019.

CORTEZ, Arnaldo Ortega. Efecto de una metodología de Aprendizaje Basado en Problemas (ABP), sobre el rendimiento académico de alumnos universitarios, AricaChile. 2018. 96f. Tese (Doctorado en Educación) — Escuela de Posgrado. Universidad Privada de Tacna. Tacna.

D’AMBRÓSIO, Ubiratan. Educação Matemática: da teoria à prática. Campinas: Papiros, 1996.

DÍAZ, Jaider Genes; POLO, Federico Nájera; TORO, Stella Monroy. Metodologías activas para la solución de problemas al enseñar Matemáticas Financieras. Omnia, v. 23, n. 1, p. 44-58, jan./abr. 2017.

DOS SANTOS, Anselmo Amaro. Intervenção educativa piloto para o ensino do pensamento crítico por docentes de Enfermagem. 2018. 246f. Tese (Doutorado em Enfermagem na Saúde do Adulto) - Escola de Enfermagem. Universidade de São Paulo. São Paulo.

DUCH, Barbara J.; GROH, Susan E.; ALLEN, Deborah E. The power of problem-based learning: a practical "how to" for teaching undergraduate courses in any discipline. Sterling: Stylus Publishing, 2001.

FLICK, Uwe. Introdução à Pesquisa Qualitativa. Tradução de José Elias Costa. Porto Alegre: Artmed, 2009.

FLOREZ, Ibeth Castro; SANDOVAL, Hugo Parra. Habilidades de pensamiento: una mirada desde la perspectiva de Angel Villarini. Omnia, v. 21, n. 3, p. 41-53, sep./dic. 2015. 
GIBBS, Grahan. El análisis de datos cualitativos en investigación cualitativa. Madrid: Ediciones Marota, 2012.

GODOY, Elenilton Vieira; ALMEIDA, Eustáquio de. A evasão nos cursos de Engenharia e a sua relação com a Matemática: uma análise a partir do COBENGE. Educação Matemática Debate, Montes Claros, v. 1, n. 3, set./dez. 2017.

GUISSO, Diego Peterle; CESCONETTO, Laisi Bellon; FIORESI, Solange Aparecida Mauro; LEITE, Ângela Maria. Aprendizagem Baseada em Problemas (PBL) no Ensino Superior: concepções introdutórias. Intelletto, Venda Nova do Imigrante, v. 4, n. 3, p. 23 29, 2019.

GUZZO, Guilherme Brambatti; LIMA, Valderez Marina do Rosário. O desenvolvimento do pensamento crítico na educação: uma meta possível? Educação Unisinos, São Leopoldo, v. 22, n. 4, p. 334-343, out./dez. 2018.

HOLGAARD, Jette Egelund; PETERSEN, Lone Stub; GUERRA, Aida; KOLMOS, Anette. Control del problema en un entorno de PBL. In: MESA, Fernando Rodríguez; KOLMOS, Anette; GUERRA, Aida. Aprendizaje basado en problemas en Ingeniería: teoria y práctica. Aalborg: Aalborg Universit Press, 2017, p. 130-167.

HUNG, Woei. The 3C3R Model: a conceptual framework for designing problems in PBL. Interdisciplinary Journal of Problem-Based Learning, v. 1, n. 1, p. 55-77, 2006.

HUNG, Woei. The 9-step problem design process for problembased learning: application of the 3C3R model. Educational Research Review, v. 4, n. 2, p. 118-141, 2009.

JERÔNIMO, Natália da Silva; ZANETTE, Eliza Netto; SCHNEIDER, Michele Domingos. A Matemática na Educação à Distância: possibilidade de uso de metodologias ativas de aprendizagem. In: SEMINÁRIO DE INTEGRAÇÃO E SOCIALIZAÇÃO DE PESQUISAS E PRÁXIS PEDAGÓGICA EM MATEMÁTICA, 7, 2019, Criciúma. Anais... Criciúma: UNESC, 2019, p. 24-27.

LIMA, Gerson Zanetta de; LINHARES, Rosa Elisa Carvalho. Escrever bons problemas. Revista Brasileira de Educação Médica, Rio de Janeiro, v. 32, n. 2, p. 197 201, abr./jun. 2008.

LIPMAN, Mathew. Critical thinking: what can it be? Educational Leadership, v. 1, n. 1, p. 38-43, 1988.

LOPES, Renato Matos; ALVES, Neila Guimarães; PIERINI, Max Fonseca; SILVA FILHO, Moacelio Veranio. Características gerais da Aprendizagem Baseada em Problemas. In: LOPES, Renato Matos, SILVA FILHO, Moacelio Veranio; ALVES, Neila Guimarães. (Org). Aprendizagem baseada em problemas: fundamentos para a aplicação no Ensino Médio e na formação de professores. Rio de Janeiro: Publiki, 2019, p. 47-74.

MANRIQUE, María Soledad; TROUSSEL. Lorena Sánchez. Más allá del pensamiento crítico: el trabajo sobre pensamento y emoción em formación docente. Didac, n. 64. p. 51-57, 2014. 
MARQUES, Ramiro. Dicionário Breve de Pedagogia. Lisboa: Editorial Presença. 2008.

MASOLA, Wilson de Jesus; ALLEVATO, Norma Suely Gomes. Dificuldade da aprendizagem Matemática: algumas reflexões. Educação Matemática Debate, Montes Claros, v. 3, n. 7, p. 52-67, jan./abr. 2019.

MAULIDIYA, Maulidiya; NURLAELAH, Ela. The effect of problem based learning on critical thinking ability in Mathematics Education. Journal of Physics: Conference Series, p. 1-4, 2019.

MENDES DA SILVA, Ivoneide; LINS, Walquiria Castelo Branco; CARNEIRO LEÃO, Marcelo Brito. Avaliação da aplicação da metodologia aprendizagem baseada em problemas na disciplina de tecnologia da informação e comunicação no ensino de Química. Educación Química, v. 30, n. 3, p. 64-78, 2019.

MORALES BUENO, Patrícia. Aprendizaje basado en problemas (ABP) y habilidades de pensamiento crítico ¿una relación vinculante? Revista Electrónica Interuniversitaria de Formación del Profesorado, v. 21, n. 2, p. 91-108. 2018.

MORÁN, José. Educação híbrida: um conceito chave para a educação de hoje. In: BACICHI, Lilian; TANZI NETO, Adolfo; TREVISANI, Fernando Mello. (Org.). Ensino híbrido: personalização e tecnologia na educação. Porto Alegre: Penso, 2015b, p. 27-45.

MORÁN, José. Mudando a educação com metodologias ativas. In: SOUZA, Carlos Alberto de; MORALES, Ofélia Elisa Torres. (Org.). Mídias Contemporâneas. Convergências Midiáticas, Educação e Cidadania: aproximações jovens. v. 2. Ponta Grossa: UEPG, 2015a, p. 15-33.

MULYANTO, Heri; GUNARHADI, Gunarhadi; INDRIAYU, Mintasih. The effect of problem based learning model on student mathematics learning outcomes viewed from critical thinking skills. International Journal of Educational Research Review, v. 3, n. 2, p. 37-45, 2018.

MUÑOZ CHOQUE, Pablo Emerson. Desarrollo de competencias financieras a través de la técnica de resolución de problemas en los estudiantes de la Facultad de Contabilidad de la Universidad de Ciencias y Humanidades (UCH). 2019. 127f. Disertación (Magister en Ciencia de la Educación) - Facultad de Contabilidad de la Universidad de Ciencias y Humanidades. Universidad Nacional de Educación Enrique Guzmán y Valle. Lima.

OLIVEIRA, Flávio Rodrigues de; COSTA, Maria Luisa Furlan; TORTORELI, Adélia Cristina; SANTOS, Ana Paula de Souza. Metodologias ativas e a Pedagogia: o ProblemBased Learning na prática curricular. Revista Aproximação, Guarapuava, v. 2, n. 3, p. 5963, 2020.

PAZÁN, Emma Georgina Gomes; FLORES, Jorge R. El Aprendizaje Basado em Problemas y el uso del Paquete Estadísdico R em la interpretación de las Gráficas de Control. Espirales, Guayaquil, v. 3, n. 26, p. 81-87, 2019.

PHILLIPS, Janet M. Strategies for active learning in online continuing education. Journal of Continuing Education in Nursing. v. 36, n. 2. p. 77-83. 2005. 
PINHO. Luis Antônio de; LOPES, Renato Matos. A construção do problema na Aprendizagem Baseada em Problemas. In: LOPES, Renato Matos; SILVA FILHO, Moacelio Veranio; ALVES, Neila Guimarães. Aprendizagem baseada em problemas: fundamentos para a aplicação no Ensino Médio e na formação de professores. Rio de Janeiro: Publiki, 2019, p. 75-116.

ROMERO, Adrian Santiago Aguinaga. Propuesta de actividades mediante la metodología ABP para la conceptualización del cálculo integral. 2019. 112f. Disertación (Magister en Docencia Matemática Universitaria) - Facultad de Ingeniería, Ciencias Físicas y Matemática. Universidad Central del Ecuador. Quito.

SIEGEL, Harvey. Educating reason: rationality, critical thinking and education. Nova York, Routledge, 1988.

SKOVSMOSE, Ole. Desafios da Educação Matemática Crítica. Tradução de Orlando de Andrade Figueiredo e Jonei Cerqueira Barbosa. Campinas: Papirus, 2008.

SKOVSMOSE, Ole. Educação Crítica: incerteza, Matemática, responsabilidade. Tradução de Maria Aparecida Viggiani Bicudo. São Paulo: Cortez, 2007.

SOUZA, Débora Vieira. O uso de problemas matemáticos no Ensino Superior sob o viés da Aprendizagem Baseada em Problemas. Revista de Educação Matemática, São Paulo. v. 16, n. 22, p. 270-283, maio/ago. 2019.

TALBERT, Robert. Flipped learning na universidade: guia para utilização da aprendizagem invertida no Ensino Superior. Tradução de Sandra Maria Mallman da Rosa. Porto Alegre: Penso, 2019.

TORP, Linda; SAGE, Sara. Problems as possibilities: Problem-Based Learning for K-16 Education. Alexandria: ACSD, 2002.

VIANA, Sidney Leandro da Silva; LOZADA, Claudia de Oliveira. Aprendizagem baseada em problemas para o ensino de probabilidade no Ensino Médio e a categorização dos erros apresentados pelos alunos. Educação Matemática Debate, Montes Claros, v. 4, n. 10 , p. $1-28,2020$.

VILLARINI JUSINO, Ángel. R. Teoría y pedagogía del pensamiento crítico. Perspectivas Psicológicas, v. 4, n. 3-4, p. 35-42, 2003.

WOOD, Daiana F. ABC of learning and teaching in Medicine: Problem-Based Learning. British Medical Journal, v. 326, n. 7384, p. 328-330, fev. 2003. 